\title{
Experimental demonstration of enhanced photon recycling in angle-restricted GaAs solar cells $\uparrow$
}

Cite this: Energy Environ. Sci., 2014, 7, 1907

\author{
Emily D. Kosten, ${ }^{a}$ Brendan M. Kayes ${ }^{b}$ and Harry A. Atwater ${ }^{\star a}$
}

Received 31st October 2013

Accepted 15th April 2014

DOI: $10.1039 / c 3 e e 43584 a$

www.rsc.org/ees

For cells near the radiative limit, optically limiting the angles of emitted light causes emitted photons to be recycled back to the cell, leading to enhancement in voltage and efficiency. While this has been understood theoretically for some time, only recently have GaAs cells reached sufficient quality for the effect to be experimentally observed. Here, as proof of concept, we demonstrate enhanced photon recycling and open-circuit voltage $\left(V_{o c}\right)$ experimentally using a narrow band dielectric multilayer angle restrictor on a high quality GaAs cell. With angle restriction we observe a clear decrease in the radiative dark current, which is consistent with the observed $V_{\text {oc }}$ increase. Furthermore, we observe larger $V_{\text {oc }}$ enhancements for cells that are closer to the radiative limit, and that more closely coupling the angle restrictor to the cell leads to greater $V_{\text {oc }}$ gains, emphasizing the optical nature of the effect.

\section{Introduction}

For ideal solar cells where all recombination is radiative, photons emitted from the cell are the sole source of carrier loss, as in the well-known Shockley-Queisser or detailed balance limit. ${ }^{1}$ Cells approaching this radiative limit have significantly higher efficiencies, as evidenced by recent world record GaAs cells, and can also exhibit new effects owing to the significant number of radiatively emitted photons. ${ }^{2-4}$ For example, optically limiting the angles of emitted light, as in Fig. 1a, causes emitted photons to be recycled back to the cell, leading to enhancement in voltage and efficiency. Despite this theoretical prediction, until recently even the highest efficiency solar cells were not close enough to the radiative limit for such an effect to be observed.$^{5-7}$ However, with the introduction of cells lifted off the growth substrate, GaAs cells have shown significant gains in efficiency due to $V_{\text {oc }}$ increases, indicating an increase in the

${ }^{a}$ Thomas J. Watson Laboratory of Applied Physics, California Institute of Technology, 1200 E. California Blvd., Pasadena, CA 91125, USA. E-mail: haa@caltech.edu

${ }^{b}$ Alta Devices, Sunnyvale, CA, USA

$\dagger$ Electronic supplementary information (ESI) available. See DOI: $10.1039 / \mathrm{c} 3 \mathrm{ee} 43584 \mathrm{a}$

\section{Broader context}

Traditionally, solar cell research focused on absorbing light and collecting the resulting carriers. In fact, only recently have III-V solar cells, particularly GaAs, achieved sufficient radiative efficiency for the light emitted from a solar cell to be a significant factor in the cell performance. As cells approach the radiative limit, where emitted light is the sole source of loss, managing the emitted light becomes a promising route to increasing efficiencies beyond the $33 \%$ detailed balance limit. Using an optic to restrict the light emission angles causes emitted photons to be recycled back to the cell and could allow for substantial efficiency and voltage increases. In fact, limiting the angles of emitted light becomes a viable flat-plate alternative to concentration for cells near the radiative limit. As a proof of concept for this approach, we use a dielectric multilayer to the restrict emission angles on a high quality GaAs cell and demonstrate a clear voltage increase due to enhanced recycling of emitted photons. We also illustrate that the voltage increase is larger for cells closer to the radiative limit, indicating that greater benefits to angle restriction are possible as solar cells continue to more closely approach this limit.

external radiative efficiency (ERE) of the cell. ${ }^{2-4}$ In these liftedoff GaAs cells radiatively emitted photons are reflected from a metallized back surface instead of being absorbed in the substrate, resulting in a large increase in ERE and $V_{\mathrm{oc}}{ }^{4,8}$ As radiative recombination is dominant in high quality GaAs, these lifted-off cells perform near the radiative limit and are therefore suitable for experimentally demonstrating enhanced photon recycling and $V_{\mathrm{oc}}$ via angle restriction of emitted light. In fact, it was recently demonstrated that a voltage increase could be observed in such cells by placing a reflecting dome above the cell to recycle emitted photons. ${ }^{9}$

Here, as proof of concept, we demonstrate enhanced photon recycling and open-circuit voltage $\left(V_{\text {oc }}\right)$ experimentally using an optical element with angle restriction only over the narrow wavelength range of emitted light in GaAs placed on a high quality GaAs cell. As in Fig. 1, we design a dielectric multilayer angle restrictor with excellent normal incidence transmission and high reflectivity at oblique angles for radiatively emitted wavelengths. Using this narrow band angle restrictor with a high quality GaAs cell, we observe enhanced photon recycling 
a) Dielectric Angle Restrictor
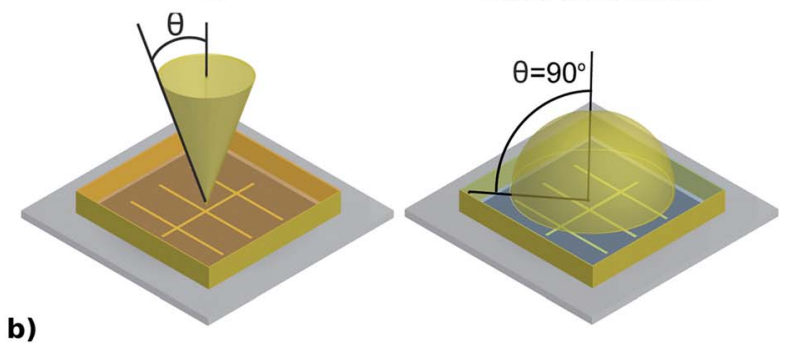

b)
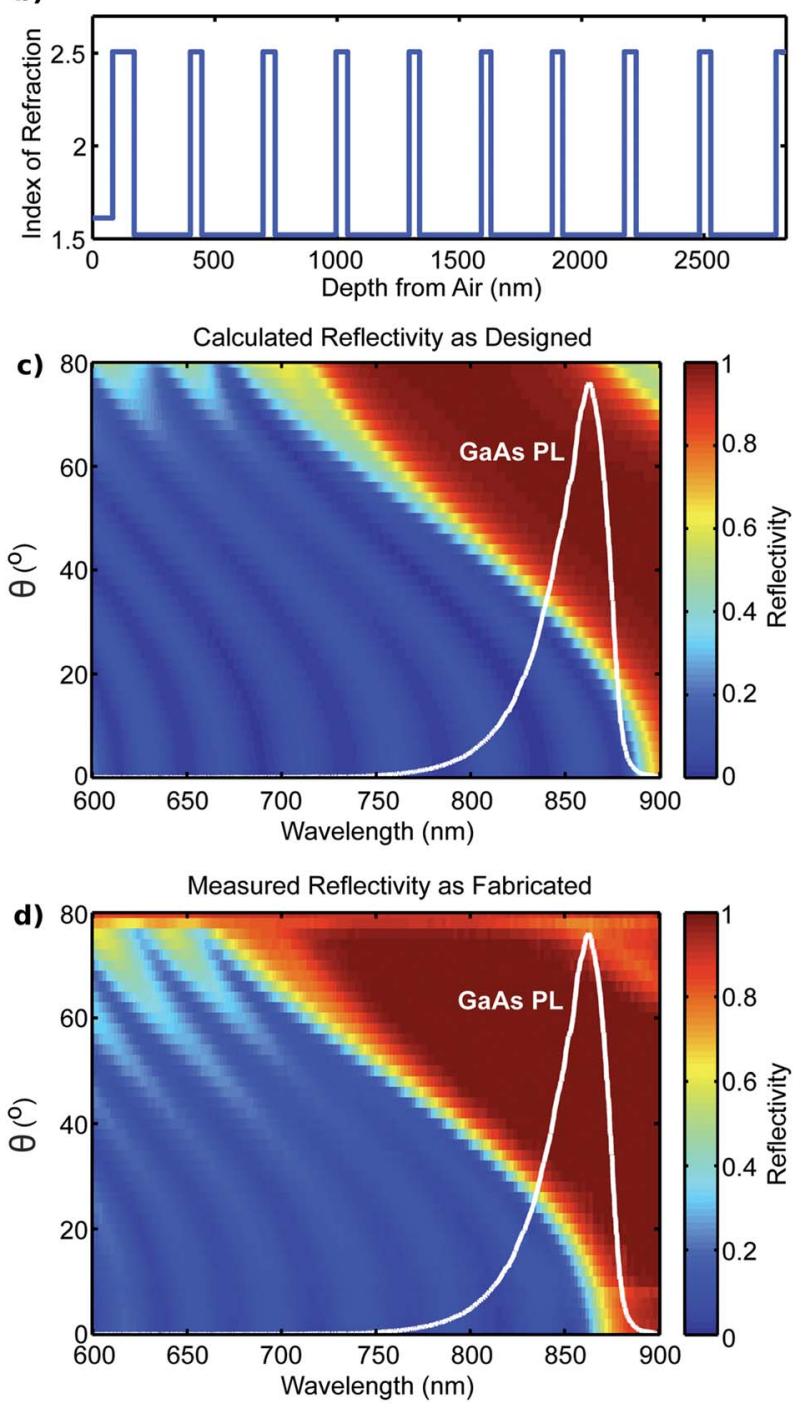

Fig. 1 Narrowband dielectric angle restrictor design and fabrication. (a) Experimental set-up: a high quality GaAs cell is placed in optical contact with either a dielectric angle restrictor deposited on fused silica (left) or a bare fused silica control (right) using fused silica index matching fluid at the GaAs-fused silica interface. The emission angles for each optic are shown schematically in yellow. For the experiments in Fig. 2 and 3, the edges of the optics are coated with a gold reflector to minimize light loss. (b) The refractive index at $800 \mathrm{~nm}$ as a function of depth for the dielectric angle restrictor multilayer design. ( 0 represents the air interface). (c) Calculated reflectivity for the dielectric angle restrictor design as a function of angle and wavelength. Angles are denoted in air, as we are only concerned with light that is not totally internally reflected. Spectrum of photoluminescence available for photon recycling (white line) indicates the wavelengths where angle restriction is desired. (d) Measured reflectivity for the dielectric angle and a resulting voltage increase. In other words, simply placing an angle restrictor on the cell causes a voltage increase of 3.6 $\mathrm{mV}$ without a change in current. In addition, we observe a $12 \%$ decrease in the radiative component of the dark current, which is consistent with the observed $V_{\text {oc }}$ increase. Considering a variety of cells, the largest $V_{\text {oc }}$ enhancements occur in cells that are closest to the radiative limit, with maximum ERE values of $15.7 \%$. Finally, we see that more closely coupling the angle restrictor to the cell leads to greater $V_{\text {oc }}$ gains, emphasizing the optical nature of the enhancement.

The predicted voltage increase from angle restriction follows directly from the principles of detailed balance. ${ }^{1}$ For a solar cell in the radiative limit at steady state and open circuit, detailed balance requires that the number of photons leaving the cell equal the number of photons entering the cell. Mathematically, we express this as

$$
\int_{E_{\mathrm{g}}}^{\infty} S(E) a(E) \mathrm{d} E=\int_{\Omega_{\mathrm{c}}} \int_{E_{\mathrm{g}}}^{\infty} a(E) \frac{2}{h^{3} c^{2}} \frac{E^{2}}{\mathrm{e}^{\left(E-q V_{\mathrm{oc}}\right) / k T}-1} \mathrm{~d} E \cos (\theta) \mathrm{d} \Omega
$$

where $a(E)$ is the fraction of photons at energy $E$ absorbed by the solar cell, $\Omega_{\mathrm{c}}$ is the solid angle the cell emits into, $S(E)$ is the solar spectrum, and $q V_{\text {oc }}$ equals the chemical potential of the cell. ${ }^{5}$ The left-hand side of the equation gives the photon flux absorbed by the cell, and the right-hand side gives the emitted photon flux at open circuit.

Assuming the $V_{\text {oc }}$ does not closely approach the bandgap, we may approximate the $V_{\text {oc }}$ under illumination as

$$
\begin{aligned}
V_{\mathrm{oc}} & \approx k T \ln \left(\frac{\int_{E_{\mathrm{g}}}^{\infty} S(E) a(E) \mathrm{d} E}{\int_{\Omega_{\mathrm{c}}} \int_{E_{\mathrm{g}}}^{\infty} a(E) \frac{2}{h^{3} c^{2}} \frac{E^{2}}{\mathrm{e}^{\left(E-q V_{\mathrm{oc}}\right) / k T}-1} \mathrm{~d} E \cos (\theta) \mathrm{d} \Omega}\right) \\
& =k T \ln \left(J_{\mathrm{sc}} / J_{0}\right)
\end{aligned}
$$

where $J_{\mathrm{sc}}$ is the short-circuit current and $J_{0}$ is the dark current, which is solely due to radiatively emitted light in the detailed balance limit. Restricting the emission angle causes photons generated by radiative recombination to be recycled and reabsorbed within the cell rather than emitted. Thus, enhanced photon recycling via angle restriction reduces $J_{0}$ and increases $V_{\mathrm{oc}}$.

For realistic cells, emitted light forms a larger fraction of $J_{0}$ in cells closer to the radiative limit. Thus, high ERE cells, like the GaAs cells in these experiments, are required for $J_{0}$ to be reduced sufficiently with angle restriction that a voltage increase may be observed. Furthermore, higher ERE cells should show larger voltage increases. For this reason, though the voltage increases in this proof-of-concept experiment are modest, further improvements in GaAs cell technology could significantly increase the performance benefits from angle restriction. In fact, for an Auger-limited GaAs cell, angle restriction is predicted to give cell efficiencies above $38 \%{ }^{6}$

restrictor as deposited with spectrum of photoluminescence available for photon recycling (white line). Reflections at the back surface of the substrate have been subtracted, see ESI. $\uparrow$ Angles are again denoted in air. For both (c) and (d), we plot only wavelengths greater than $600 \mathrm{~nm}$, to correspond with the wavelengths of illumination in the experiment. 
For terrestrial applications, we envision a flat plate, one sun, angle restricting system with high quality GaAs cells. While tracking may be beneficial, high accuracy tracking is not required as dielectric angle restrictors have a relatively large acceptance angle. Furthermore, for cells in the ERE range considered here, narrow angle restriction has limited benefit, as non-radiative recombination limits the possible voltage increase. (The ESI $\dagger$ includes a calculation with the angle dependence of $J_{\mathrm{sc}}$.) Recent work has demonstrated the fabrication of high ERE cells in other III-V materials, notably GaInP, suggesting that this approach will become more broadly applicable with continued cell development, and could easily be incorporated with multijunctions. ${ }^{10}$ Use with these cell technologies also suggests early applications in military and space solar, where efficiency and weight are paramount.

While our previous work has considered broadband ray optical angle restrictors with light trapping cells, the cells in this experiment have high reflectivity specular back reflectors that are metallic, with reflectivity of $75.5 \%$, or metallodielectric, with reflectivity of $99.7 \%$. The calculated reflectivity values refer to band edge $(873 \mathrm{~nm})$ emission angle-averaged within the GaAs. As the solar cells are planar and do not incorporate light trapping, we utilize a dielectric multilayer that provides angle restriction only over the narrow range of wavelengths at the semiconductor band edge where the GaAs cells emit light (see Fig. 1). This narrowband angle restriction allows diffuse, non-normal incidence light to enter over most of the spectral range (see ESI $\dagger$ ). Capturing this diffuse light gives significant current enhancements relative to a broadband concentrator or angle restrictor. In addition, potential losses due to tracking errors are greatly reduced, and simpler, cheaper trackers may be utilized. As in the ESI, $\dagger$ we envision depositing such an angle restrictor in place of an anti-reflective coating, so the cost derives only from the added layers relative to a conventional AR coat.

As shown in Fig. 1b, the angle restrictor design consists of alternating high and low index layers with large refractive index contrast to increase the angular range of reflection. ${ }^{\mathbf{1 1 - 1 3}}$ While the design is not strictly periodic, the angular properties can be understood from the Bragg condition

$$
\cos \theta=\frac{m \lambda}{2 \Gamma}
$$

where $\theta$ is the angle of maximum reflectivity, $\lambda$ is the wavelength, $\Gamma$ is the period of the multilayer, and $m$ is an integer. ${ }^{\mathbf{1 4}}$ For shorter wavelengths maximum reflectivity occurs away from normal incidence, providing angle restriction for emitted light and excellent transmission at normal incidence in both the designed and fabricated structures. We note that total internal reflection owing to the high index of GaAs already provides significant photon recycling within the cell, and despite this, there is still a substantial loss due to emitted light, as ERE estimates indicate. ${ }^{4}$ As the measured reflectivity in air, see Fig. 1d, demonstrates, the dielectric structure provides photon recycling of light that would otherwise be emitted. This enhanced photon recycling occurs in addition to the photon
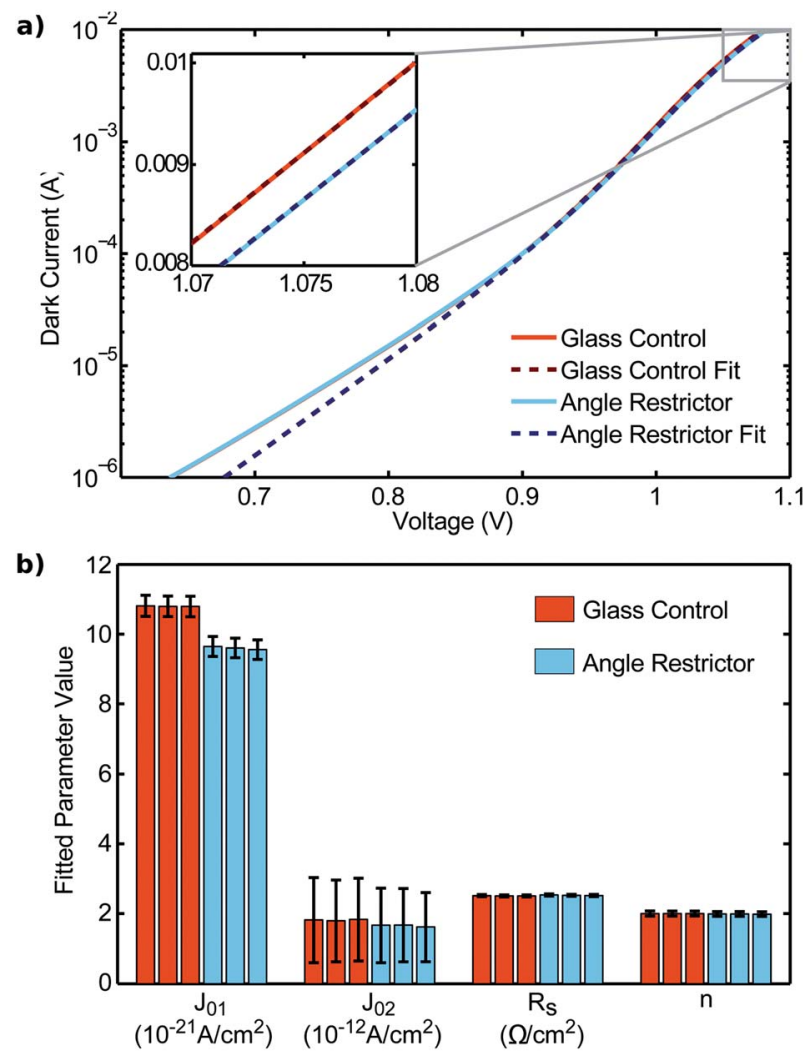

Fig. 2 Dark current measurements and fits. (a) Representative dark current curves and double diode fits for both the angle restrictor and control cases. Inset: a clear reduction in dark current is evident near $V_{\text {oc }}$ for the angle restrictor case. (b) Double diode fitted parameter results with each bar representing one of three trials on the same 15.7\% ERE cell for both the angle restrictor and fused silica control. The error bars represent $95 \%$ confidence intervals derived from the fit. Consistent with reduced radiative loss, $J_{01}$ shows a marked decrease with angle restriction while all other parameters remain unchanged.

recycling via total internal reflection, which is unaffected by the dielectric structure.

\section{Results and discussion}

Theory clearly indicates that enhanced photon recycling via angle restriction will result in a reduction of the radiative dark current. We therefore measured the dark current characteristics of a single cell under both the angle restrictor and a bare fused silica control optic, as in Fig. 1a, with fused silica index matching fluid at the interface of the cell and the fused silica substrate to avoid extraneous reflections. In the high voltage region near $V_{\text {oc }}$, where radiative emission contributes most significantly to the dark current, we see a clear decrease in dark current with angle restriction, as in Fig. 2a. To quantify this we fit the dark current, $J_{0}$, over the high voltage 0.6 to $1.1 \mathrm{~V}$ region, to the double diode equation

$$
J_{0}=J_{01}\left(\mathrm{e}^{\frac{q\left[V-J_{\mathrm{dark}} R_{\mathrm{s}}\right]}{k T}}-1\right)+J_{02}\left(\mathrm{e}^{\frac{q\left[V-J_{\mathrm{dark}} R_{\mathrm{s}}\right]}{n k T}}-1\right)
$$


where $J_{01}$ is the high voltage dark current component, $J_{02}$ is the low voltage component, $R_{\mathrm{S}}$ is the series resistance, and $n$ is the diode ideality factor. ${ }^{15,16}$ For both the control and angle restriction curves, the fit is excellent over several orders of magnitude. The fit deviates somewhat at very low currents, which we attribute to shunt resistance and has been previously observed in similar cells. ${ }^{2}$ As Fig. $2 \mathrm{~b}$ shows, $J_{02}, R_{\mathrm{s}}$ and $n$ are unchanged with angle restriction and $n$ is very close to two, indicating that the double diode model is valid. ${ }^{15,16}$ In contrast, the $J_{01}$ term, which has the same voltage dependence as radiative recombination, shows a $12 \%$ decrease with angle restriction, well beyond the error of the fit. Thus, by simply changing the optic above the cell to an angle restrictor, we observe a definite reduction in the dark current. Specifically, the reduction occurs in the high voltage dark current component attributable to radiative loss, indicating that angle restriction is enhancing photon recycling within the cell.

In addition to a reduction in dark current, we also expect a direct $V_{\text {oc }}$ enhancement under illumination. Furthermore, this voltage enhancement should be larger for cells with higher ERE, as more photons are available to be recycled via angle restriction. We therefore measured light current-voltage curves for a set of four cells with differing back reflector and material quality leading to significant variations in ERE across the cells, as determined from the $J_{\mathrm{sc}}$ and $V_{\mathrm{oc}}$ characteristics under the control optic (see ESI† for further model details). Owing to a reflecting band in the optical coupler around $550 \mathrm{~nm}$, we limited the spectrum in this proof-of-concept experiment to wavelengths longer than $605 \mathrm{~nm}$. (As in the ESI, $\uparrow$ this reflecting band can be eliminated with a rugate filter optical design, but for the initial coupler we did not pursue these structures as they are more difficult to fabricate. ${ }^{17,18}$ ) As shown in Fig. 3a, when we directly compare the control and angle restrictor on the same cell, current losses of 3.5 to $5.3 \%$ are observed with angle restriction, consistent with the measured normal incidence reflectivity of the angle restrictor. Without a change in the dark current, a reduction in $J_{\mathrm{sc}}$ would normally produce a corresponding reduction in $V_{\mathrm{oc}}$, as in eqn (1). However, $V_{\mathrm{oc}}$ increases of up to $2.5 \mathrm{mV}$ are observed under angle restriction for the highest ERE cells, as dark current reduction is the dominant effect. Thus, angle restriction increases cell voltage without any change in the illumination, and despite a reduction in $J_{\mathrm{sc}}$. Furthermore, as we expect for photon recycling, the voltage change tracks the cell ERE.

Fortunately, these current losses are not intrinsic, and result from the simplicity of our initial angle restrictor design ${ }^{\mathbf{1 7 , 1 8}}$ (see ESI $\dagger$ ). To isolate the photon recycling effect, we adjust the solar simulator to equalize the currents between the control and angle restrictor, as in Fig. $3 \mathrm{~b}$. Once $J_{\mathrm{sc}}$ values are matched for the angle restrictor and control, voltage increases ranging from $1.2 \mathrm{mV}$ to $3.6 \mathrm{mV}$ are seen for all cells, with higher ERE cells showing larger voltage increases. As the $15.7 \%$ ERE cell was also used for dark current measurements, we can compare the change in $J_{01}$ to the observed change in $V_{\mathrm{oc}}$. Since $V_{\mathrm{oc}}=$ $k T \ln \left(J_{\text {sc }} / J_{0}\right)$ and the $J_{01}$ term is dominant near $V_{\text {oc }}$, the change in $V_{\text {oc }}$ should be approximately $k T \ln \left(J_{01} / J_{01}{ }^{\prime}\right)$, where $J_{01}{ }^{\prime}$ indicates the average fitted value with the angle restrictor. Using this a)

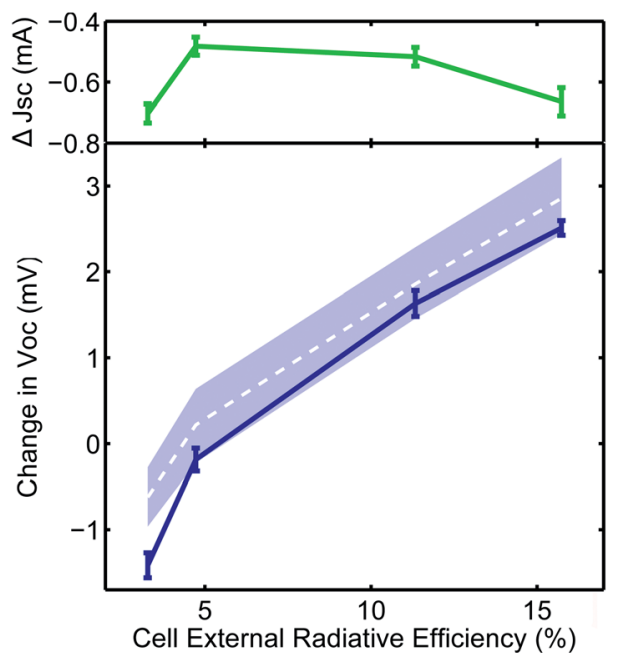

b)

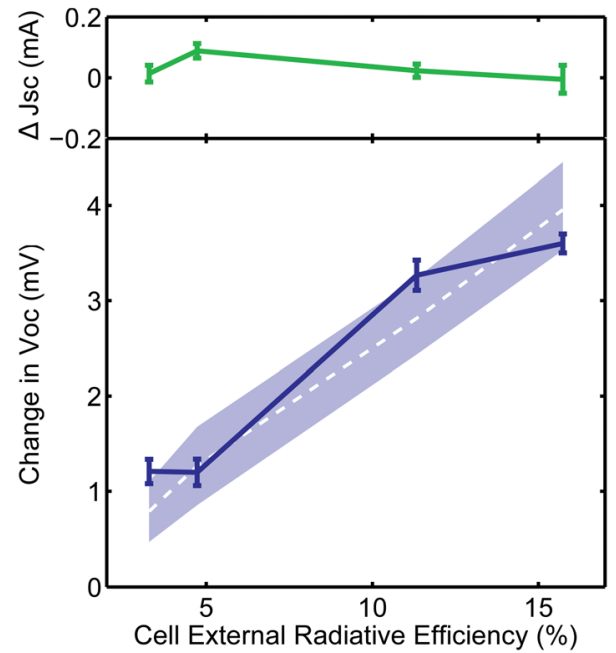

Fig. 3 Voltage increase as a function of radiative efficiency. Measured changes in open-circuit voltage (dark blue line) and short-circuit current (green line) with angle restriction. The set of four cells is plotted as a function of external radiative efficiency (ERE) determined from $J_{\mathrm{Sc}}$ and $V_{\mathrm{OC}}$ under the control optic. Variations in ERE occur between cells owing to differences in back reflectors and material quality. The error bars are calculated from standard deviation of five measured trials. The dotted white line indicates the expected voltage increase based on a modified detailed balance calculation. The light blue area shows the expected range of the model based on uncertainty in $J_{\mathrm{sC}}, V_{\mathrm{oc}}$, and temperature. (a) No solar simulator adjustment. Cells with high external radiative efficiency show a voltage increase despite a reduction in current. (b) The solar simulator was adjusted so that currents were equalized with the angle restrictor and control. With this current equalization, all cells see a voltage increase, with high ERE cells seeing a larger voltage increase.

approach, we predict from the dark current fits that the $V_{\mathrm{oc}}$ increase should be $3.0 \mathrm{mV}$, which is reasonably consistent with the measured value of $3.6 \mathrm{mV}$ for this cell. Thus, we observe a clear $V_{\mathrm{oc}}$ increase with angle restriction that is consistent with our dark current measurements, and an ERE trend that indicates enhanced photon recycling as the mechanism.

We also develop a model that directly relates the voltage increase to the optical characteristics of the angle restrictor. While detailed balance is traditionally considered an idealized 


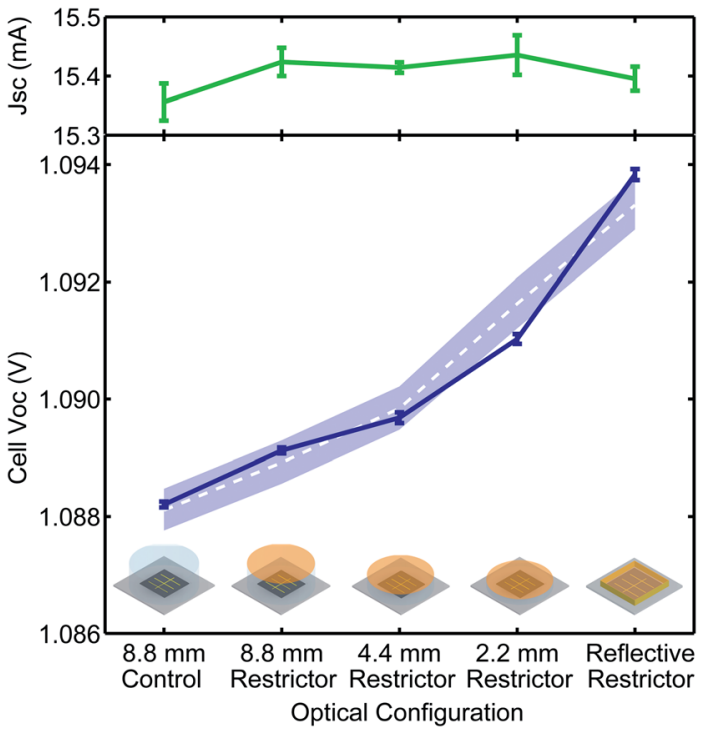

Fig. 4 Voltage increase as a function angle restrictor coupling. Measured open-circuit voltage (dark blue line) and short-circuit current (green line) as angle restriction is increased by coupling the angle restrictor more closely to the $15.7 \%$ ERE cell. The $x$-axis labels indicate the height of the fused silica spacer below the dielectric angle restrictor, or bare fused silica control, as on the far left. For all but the rightmost optical configuration, the sides of the fused silica spacers are uncoated to so emitted light may escape. Thus, a taller spacer allows more light to escape from the sides, reducing photon recycling to the 1 $\mathrm{cm}^{2}$ cell. In the rightmost configuration, the sides of the fused silica substrate are coated with a reflector to avoid side loss and maximize photon recycling by the dielectric angle restrictor. The error bars are calculated from standard deviation of five measured trials. The dotted white line indicates the expected $V_{\text {oc }}$ based on a modified detailed balance calculation. The light blue area shows the expected range of the model based on uncertainty in $J_{\mathrm{sc}}, V_{\mathrm{oc}}$, and temperature. The solar simulator was adjusted as necessary to equalize the currents across the various optical configurations.

model, we have developed a more realistic detailed balance model that includes the cell thickness, anti-reflective coating, back reflectors, and Auger and surface recombination (further details are provided in the ESI $\dagger$ ). To account for the optical environment, we calculate the angle-averaged emissivity for both the control and the angle restrictor based on measured reflectivity data as in Fig. 1d. For each cell, we use the values for $J_{\mathrm{sc}}$ and $V_{\mathrm{oc}}$ measured under the control optic to determine the cell's ERE (see ESI $\dagger$ ). We then predict the $V_{\text {oc }}$ under angle restriction based on the previously determined ERE and measured $J_{\mathrm{sc}}$. Finally, the observed temperature fluctuations of $0.1{ }^{\circ} \mathrm{C}$ and uncertainty estimates for $J_{\mathrm{sc}}$ and $V_{\mathrm{oc}}$ are used to determine the range of the prediction, as in Fig. 3. These calculations agree quite well with the experimental results, indicating that the reduction in emissivity with angle restriction and the resulting photon recycling enhancement fully explain the observed differences in $V_{\text {oc}}$.

Lastly, we perform a series of experiments where we gradually increase the photon recycling and $V_{\text {oc }}$ by coupling the angle restrictor more closely to the cell. As shown in Fig. 4, we begin by placing a large, uncoated fused silica cylinder above the cell which allows light to escape unimpeded from both the sides and the top of the cylinder. In essence, this fused silica spacer facilitates the outcoupling of light emitted from the solar cell to free space, similar to the glass sphere often used with lightemitting diodes. As before, index matching fluid is used at the fused silica-GaAs cell interface. Then, the angle restrictor is placed on a series of fused silica spacers with non-reflecting, uncoated sides that allow light to escape, with index matching fluid between the spacer and the angle restrictor substrate. As the height of the spacer is reduced, less light escapes through the transparent sides of the spacers and more light is recycled back to the cell by the dielectric angle restrictor. Finally, we use an angle restrictor with reflecting sides to prevent light escape from the sides of the fused silica substrate and maximize the photon recycling. As Fig. 4 illustrates, more closely coupling the angle restrictor to the solar cell increases the observed $V_{\mathrm{oc}}$, demonstrating that more effective angle restriction leads to enhanced photon recycling and $V_{\text {oc }}$. We also find close agreement between the experiment and realistic detailed balance calculations, indicating that the coupling of the angle restrictor explains the observed changes in $V_{\text {oc }}$.

\section{Conclusion}

We have performed a series of experiments that clearly demonstrate enhanced photon recycling and resulting $V_{\mathrm{oc}}$ increases of up to $3.6 \mathrm{mV}$ via angle restriction with a narrowband dielectric multilayer angle restrictor. Dark current measurements show a $12 \%$ decrease in the radiative component of the dark current consistent with the observed voltage enhancement. In addition, measurements of the voltage increase on several cells illustrate that cells closer to the radiative limit show larger voltage enhancements, as we expect for photon recycling. These measurements also show good agreement with calculations based on the measured reflectivity of the angle restrictor. Finally, we have shown that more closely coupling the angle restrictor to the cell leads to predictable increases in voltage for several configurations, emphasizing that this voltage increase is due to a purely optical photon recycling effect.

Thus, we have demonstrated as a proof of concept that angle restriction with a narrowband dielectric multilayer leads to enhanced photon recycling and a corresponding voltage increase in high quality GaAs cells. The narrowband angle restrictor approach has significant advantages in admitting diffuse light and in the relatively simple design that can replace an existing anti-reflective coating. While the voltage enhancements shown here are relatively small, the effect becomes much larger as ERE increases and cells approach the radiative limit. High ERE cells are already being developed for III-V materials to achieve the highest possible voltage and efficiency, and these cells are ideal candidates for a broader applicability of the angle restriction approach. ${ }^{\mathbf{1 0}}$ As further improvements are made in III-V cell technology and other materials reach the high ERE regime, this approach holds promise for significantly increasing cell efficiencies in a flat plate geometry. 


\section{Acknowledgements}

Thanks to M. Sheldon, E. Warmann, J. Bosco, and C. Eisler for useful discussions and comments and to J. Lloyd for assistance with figure preparation. This work was supported by the the 'Light-Material Interactions in Energy Conversion' Energy Frontier Research Center funded by the U.S. Department of Energy, Office of Science, Office of Basic Energy Sciences under Award Number DE-SC0001293. E.K. is also the recipient of a Resnick Sustainability Institute Graduate Fellowship.

\section{References}

1 W. Shockley and H. J. Queisser, Detailed balance limit of efficiency of p-n junction solar cells, J. Appl. Phys., 1961, 32(3), 510-519.

2 B. M. Kayes, H. Nie, R. Twist, S. G. Spruytte, F. Reinhardt, I. C. Kizilyalli and G. S. Higashi, $27.6 \%$ conversion efficiency, a new record for single-junction solar cells under 1 sun illumination, Proceedings of 37th IEEE Photovoltaic Specialists Conference, 2011, pp. 4-8.

3 M. A. Steiner, J. F. Geisz, I. García, D. J. Freidman, A. Duda and S. R. Kurtz, Optical enhancement of the open-circuit voltage in high quality GaAs solar cells, J. Appl. Phys., 2013, 113, 123109.

4 M. A. Green, Radiative efficiency of state-of-the-art photovoltaic cells, Prog. Photovoltaics, 2012, 20(4), 472-476.

5 A. Martí, J. L. Balenzategui and R. F. Reyna, Photon recycling and Shockely's diode equation, J. Appl. Phys., 1997, 82(8), 4067-4075.

6 E. D. Kosten, J. H. Atwater, J. Parsons, A. Polman and H. A. Atwater, Highly efficient GaAs solar cells by limiting light emission angle, Light: Sci. Appl., 2013, 2, e45.

7 J. N. Munday, The effect of photonic bandgap materials on the shockley-queisser limit, J. Appl. Phys., 2012, 112(6), 064501.

8 O. D. Miller, E. Yablonovitch and S. R. Kurtz, Strong internal and external luminescence as solar cells approach the
Shockley-Queisser limit, IEEE Journal of Photovoltaics, 2012, 2, 303-311.

9 A. Braun, E. A. Katz, D. Feuermann, B. M. Kayes and J. M. Gordon, Photovoltaic performance enhancement by external recycling of photon emission, Energy Environ. Sci., 2013, 6, 1499-1503.

10 J. F. Geisz, M. A. Steiner, I. García, S. R. Kurtz and D. J. Freidman, Enhanced external radiative efficiency for 20.8\% efficient single-junction GaInP solar cells, Appl. Phys. Lett., 2013, 103(4), 041118.

11 M. Deopura, C. K. Ullal, B. Temelkuran and Y. Fink, Dielectric omnidirectional visible reflector, Opt. Lett., 2001, 26(15), 1197-1199.

12 Y. Fink, S. Winn, J. N. ad Fan, C. Chen, J. Michel, J. D. Joannopoulos and E. L. Thomas, A dielectric omnidirectional reflector, Science, 1998, 282, 1679-1682.

13 E. Yablonovitch, Engineered omnidirectional externalreflectivity spectra from one-dimensional layered interference filters, Opt. Lett., 1998, 23(21), 1648-1649.

14 B. E. A. Saleh and M. C. Teich, Fundamentals of Photonics, Wiley, 2nd edn, 2007.

15 S. P. Tobin, S. M. Vernon, C. Bajgar, S. J. Wojtczuk, M. R. Melloch, A. Keshavarzi, T. B. Stellwag, S. Venkatensan, M. S. Lundstrom and K. A. Emery, Assessment of MOCVD- and MBE-grown GaAs for highefficiency solar cell applications, IEEE Trans. Electron Devices, 1990, 37(2), 469-477.

16 M. T. Sheldon, C. N. Eisler and H. A. Atwater, GaAs passivation with trioctylphosphine sulfide for enhanced solar cell efficiency and durability, Adv. Energy Mater., 2012, 2(3), 339-344.

17 S. Fahr, C. Ulbrich, T. Kirchartz, U. Rau, C. Rockstuhl and F. Lederer, Rugate filter for light-trapping in solar cells, Opt. Express, 2008, 16(13), 9332-9343.

18 C. Ulbrich, S. Fahr, J. Upping, M. Peters, T. Kirchartz, C. Rockstuhl, R. Wehrspohn, A. Gombert, F. Lederer and U. Rau, Directional selectivity and ultra-light-trapping in solar cells, Phys. Status Solidi A, 2008, 205(12), 2831-2843. 